\title{
Overview of theoretical studies of Rashba effect in polar perovskite surfaces
}

\author{
K. V. Shanavas \\ Department of Physics, University of Missouri, Columbia, Missouri 65211, USA \\ Oak Ridge National Laboratory, 1 Bethel Valley Road, Tennessee 37831, USA
}

\begin{abstract}
Theoretical studies with the help of first-principles electronic structure calculations and tight-binding based Hamiltonian models aimed to understand the Rashba effect in the 2D electron gas at the surfaces and interfaces of polar perovskite oxides are discussed. First-principles calculations on a slab of $\mathrm{KTaO}_{3}$ show that the spin-splitting is orbital dependent and is greatly suppressed by the lattice relaxation close to the surface. However, the electron gas is amenable to tuning by external potentials perpendicular to the surface and can be used to control Rashba splitting. Construction of a minimal model Hamiltonian to study $d$ orbitals under uniform electric field is explained. The potential introduces new matrix elements between orbitals by breaking the symmetry and distorting the lattice. When coupled with spin-orbit interaction, this results in lifting the spin degeneracy.
\end{abstract}

\section{Introduction}

The momentum-dependent spin splitting in a two dimensional electron gas (2DEG) subjected to a perpendicular electric field, known as the Rashba effect, [1] has been observed in numerous condensed matter systems including metal surfaces, $[2,3]$ topological insulators $[4,5]$ and interfaces and surfaces of perovskite oxides. $[6,7,8,9]$ Potential applications in spintronics devices [10]

Email address: kavungalvees@ornl.gov (K. V. Shanavas)

Preprint submitted to Elsevier

July 6, 2014 
has led to many theoretical studies aimed to understand the origin and ways to control the Rashba effect.

The electrons moving near the surface see the potential gradient as a magnetic field, which couples with the spin. For nearly free electrons with parabolic band dispersion, this effect can be described by the famous Bychkov-Rashba Hamiltonian, [11] which has the form $H_{R}=\alpha_{R}\left(k_{y} \sigma_{x}-k_{x} \sigma_{y}\right)$. Here, $\left(k_{x}, k_{y}\right)$ are components of the electron crystal momentum, $\left(\sigma_{x}, \sigma_{y}\right)$ are the Pauli matrices and $\alpha_{R}$ is the Rashba parameter. The electrons are confined in the $x y$ plane and the electric field is along $z$ direction. It leads to a linear spin splitting in the band structure, of the form, $\varepsilon_{k}=\hbar^{2} k^{2} / 2 m^{*} \pm \alpha_{R} k$ as shown in Fig. 1. For electrons in the solid, $\alpha_{R}$ depends on the type of orbitals involved, the screened electric field and the strength of spin-orbit coupling.

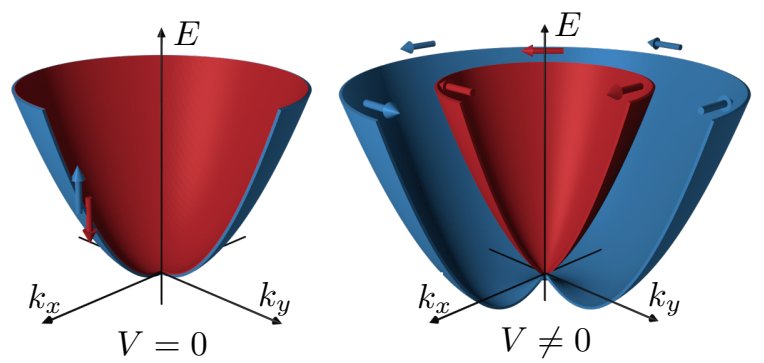

Figure 1: Energy levels of 2DEG are parabolic $E \propto k^{2}$ and are spin-degenerate in the presence of inversion and time reversal symmetries $\left(E_{\uparrow}=E_{\downarrow}\right)$ as shown in the left side. An external electrostatic potential $(V \neq 0)$ perpendicular to the plane breaks the inversion symmetry leading to the Rashba splitting of levels (right). The spins are now momentum dependent due to spin-orbit coupling.

From device applications point of view, the interfaces and surfaces of polar perovskite oxides of the type $\mathrm{ABO}_{3}$ offer an excellent system to study the Rashba effect. The alternating charges on $\mathrm{AO}$ and $\mathrm{BO}_{2}$ lead to a diverging potential or "polar catastrophe", [12] which can be compensated by $1 / 2$ electron per unitcell at the surface. A 2DEG is thus formed, either via electronic reconstruction, [13] oxygen vacancies [14] or interdiffusion of atoms [15] or a combination of them. [16] The 2DEG has high mobility parallel to the surface but is strongly localized perpendicular to it. In addition, many of these ma- 
terials have high $Z$ elements with strong spin-orbit coupling, which is another necessary ingredient for Rashba splitting. Experiments have found evidence of Rashba effect at the $\mathrm{LaAlO}_{3} / \mathrm{SrTiO}_{3}$ interface, $[7,6]$ with an asymmetric dependence on the sign of the electric field applied along the interface normal. A subband structure and sizable spin-orbit interaction were observed at the surfaces of $\mathrm{KTaO}_{3}$ in photoelectron spectroscopy measurements. [9]

Theoretical methods such as first-principles calculations based on density functional theory (DFT) and tight-binding (TB) models have been successful in understanding the Rashba effect in polar perovskite systems. [17, 18, 19, 20, 21] In TB models, the effect of broken inversion symmetry is taken into account through field dependent orbital overlap parameters. For example, in the case of $p$ orbitals on a plane, without any external perturbations the inter-site couplings between $p_{x}, p_{y}$ orbitals and $p_{z}$ orbitals are zero by symmetry. The broken symmetry results in nonzero overlap between these states, which in the presence of spin-orbit interaction lead to splitting of up and down spin bands away from $\Gamma$ point. [22] Model studies on $s p$-band systems, such as graphene, have shown that in addition to these intersite coupling terms [23] there are also onsite terms because the orbitals are no longer orthogonal. [24] For $d$ orbital systems, first principles calculations [20] and TB models $[17,18]$ show good agreement with experimental observations. In these systems, the lattice distortions caused by the polar field also contribute towards Rashba splitting. [19] On the other hand, the lattice relaxation near the surface can significantly weaken the effect. [20] Rashba effect is also suggested to result in an instability towards phase separation of the electron gas at the interface. [25]

An overview of the theoretical studies on the Rashba spin-orbit coupling effects on the 2DEG band dispersions is presented in this manuscript. Taking the $\mathrm{KTaO}_{3}$ as a typical example of a polar surface, results of DFT calculations are summarized in the next section. Steps to construct a TB model Hamiltonian is discussed in the section following it. 


\section{DFT calculations}

The electronic structure calculations are performed using ab-initio density functional theory as implemented in the Vienna ab-initio simulation package. [26, 27] Computational methods and details of the calculations are discussed elsewhere. [20] The results discussed in this section are for calculations on an (001) slab of $\mathrm{KTaO}_{3}$, but should be applicable to a wider range of perovskite systems.

\subsection{Electronic structure}

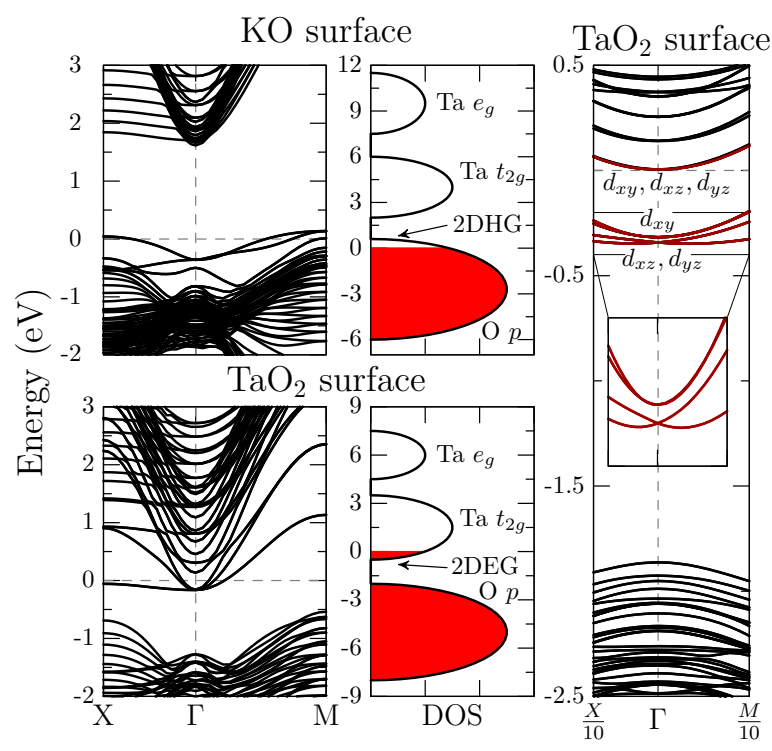

Figure 2: DFT band structures for the $\mathrm{KO}$ (top) and $\mathrm{TaO}_{2}$ (bottom) terminated surfaces of ideal unrelaxed structures. Sketch of the resultant density of states (DOS) for the two cases are shown in the middle panels. A $2 \mathrm{D}$ electron gas forms in the Ta- $t_{2 g}$ states when the surface is $\mathrm{TaO}_{2}$ terminated. In the right panel, energy bands around $\Gamma$ point in the presence of spin-orbit coupling is shown. The bands that exhibit largest Rashba splitting are marked in red. The inset zooms in to show the effect more clearly.

In the bulk form, $\mathrm{KTaO}_{3}$ is insulating with $\mathrm{O}-p$ states in the valence band and Ta- $d$ in the conduction band. A large crystal field splits the $d$ manifold into $e_{g}$ and $t_{2 g}$ levels as shown in Fig. 2. The structure consists of alternating $\mathrm{KO}^{+}$and $\mathrm{TaO}_{2}{ }^{-}$layers, so in the calculations, it is possible to form either an electron gas (2DEG) or a hole gas (2DHG) at the surface by terminating the slab by eitherTaO $\mathrm{Ta}_{2}$ or $\mathrm{KO}$ layers respectively. 
From the layer resolved electron density we see that the 2DEG (or 2DHG) is confined to a few unitcells close to the surface. These states feel the intrinsic potential and when spin-orbit coupling is included in the calculation, the spin degeneracy is lifted by the Rashba effect (Right panel of Fig. 2). The amount of splitting depends on the orbital character of the bands in addition to the strength of the spin-orbit coupling. As shown in Fig. 2, the bands that exhibit strong Rashba splitting have predominant Ta $d_{x z}+d_{y z}$ character.

\subsection{Structure of $\mathrm{TaO}_{2}$ surface}
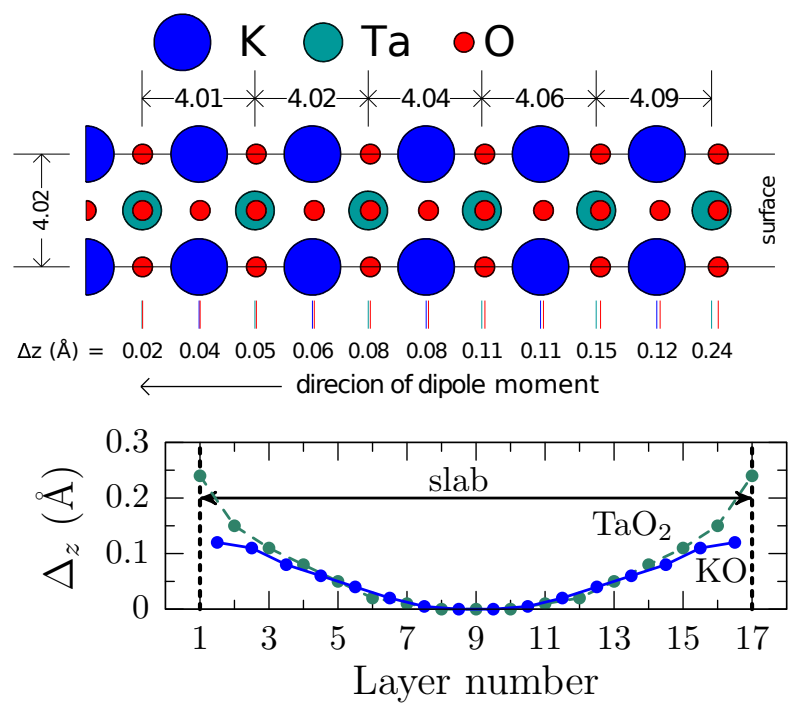

Figure 3: Structural relaxation of the $\mathrm{KTaO}_{3}$ slab near the surface. Side view of the structure in the $a c$ plane is shown in the top panel. The lower panel shows the change of the cationanion distances along $z$ in various layers due to relaxation. The slab is symmetric, with layers No. 1 and 17 being the two surface $\mathrm{TaO}_{2}$ layers.

Experimental measurements on $\mathrm{KTaO}_{3}$ observed subband structures similar to what is shown in Fig. 2, but could not resolve any spin-splitting despite the presence of heavy Ta ions with strong spin-orbit coupling. [9] Relaxation induced structural distortions shown in Fig. 3 are probably responsible for diminishing the Rashba splitting in $\mathrm{KTaO}_{3}$. [20] The structural relaxation results in oxygen atoms at the surface to shift out of the Ta plane by as much as $0.24 \AA$, producing a local dipole moment pointing in the opposite direction to the polar field. The 
distortions decay rapidly and the ionic positions approach their bulk values within about six KTO layers. However, they weaken the polar field and as a consequence, the 2DEG which was confined to within $3 \mathrm{TaO}_{2}$ layers in the unrelaxed structure spreads to about 8 layers as shown in the top panel of Fig. 4. Since, most of the contribution to the Rashba splitting comes from the top layers ( $70 \%$ from first layer, $20 \%$ from second layer, $5 \%$ from third layer and so on), structural relaxation significantly reduces the Rashba splitting. The distortions also raises the energy of the states with $d_{x z}+d_{y z}$ character near the surface, effectively moving those electrons deeper into the bulk. Since these states contribute most to the splitting, as can be seen in Fig. 2, this further diminishes the splitting.

Effect of an external potential on the distribution of 2DEG near the surface of an optimized $\mathrm{KTaO}_{3}$ slab is shown in Fig. 4. Any applied field will be partially screened by the lattice, but as Fig. 4 shows, a field of $0.12 \mathrm{eV} / \AA$ can move both type of carriers closer to the surface by about $5 \AA$. As a consequence, the average Rashba parameter $\alpha_{R}$ is doubled from $0.015 \mathrm{eV} / \AA$ to $0.032 \mathrm{eV} / \AA$. Even though typical experimental fields are smaller (few hundred volts across a micrometer of sample thickness), can still detect the changes. [6]

\section{Model Hamiltonian}

A parametrized model Hamiltonian that fit the DFT band structure is useful in understanding the microscopic origin of different features and by varying the parameters we can see what roles they play. We use the tight-binding framework with atomic orbitals as basis functions. For the lattice, only the surface layer is considered with the broken symmetry incorporated via onsite energies of the orbitals and an external potential gradient perpendicular to the plane.

\subsection{Energy levels at the surface}

An understanding of the symmetry and energy levels of the system is necessary to proceed further. Under the cubic $\left(O_{h}\right)$ field, the five $d$ orbitals split into two-fold $\Gamma_{12}$ levels $\left(e_{g}: d_{z^{2}}, d_{x^{2}-y^{2}}\right)$ and three-fold $\Gamma_{25}^{\prime}$ levels $\left(t_{2 g}: d_{x y}, d_{y z}, d_{x z}\right)$ 


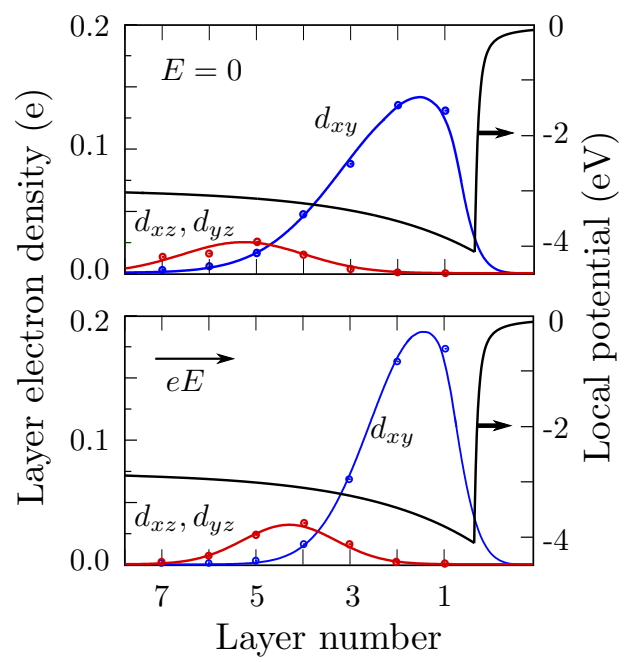

Figure 4: DFT calculated average local potentials (black line) and 2DEG charge density envelope function for $d_{x y}$ (blue) and $d_{x z}+d_{y z}$ (red) for relaxed $\mathrm{KTaO}_{3}$ slabs. For the bottom panel a potential gradient of $0.12 \mathrm{~V} / \AA$ is applied such that $e \vec{E}$ points towards the surface. Layer number 1 correspond to the surface $\mathrm{TaO}_{2}$.

separated by $\Delta$. Spin-orbit coupling further breaks the $t_{2 g}$ states into a two-fold $\Gamma_{7}^{+}$and a four-fold $\Gamma_{8}^{+}$separated by $3 \lambda / 2 .[28]$ The $e_{g}$ states remain degenerate and have symmetry $\Gamma_{8}^{+}$, as shown in Fig. 5 .

When a surface is formed, the symmetry is reduced to $C_{4 v}$ and the $e_{g}$ states and the degenerate $\Gamma_{8}^{+}$states in $t_{2 g}$ split into $\Gamma_{6}$ and $\Gamma_{7}$ by $\delta$ and $\epsilon$ respectively leading to five distinct levels. [20] At $\Gamma$ point, the interaction between $\Gamma_{7}$ and $\Gamma_{6}$ levels are forbidden by symmetry, although they are alowed to interact with other levels within their group. All levels are spin-degenerate at when $k=0$, but show $k$ dependent splitting away from it.

\subsection{Tight-binding model}

The Hamiltonian can be separated into three components; arising from electron hopping, spin-orbit coupling and electrostatic potential:

$$
H=H_{\mathrm{K}}+H_{\mathrm{SO}}+H_{\mathrm{E}} .
$$

The $\mathrm{TaO}_{2}$ surface layer consists of Ta ions interconnected through oxygens in the plane and linked to $\mathrm{K}$ ions through $\mathrm{O}$ below the surface. Since 2DEG 


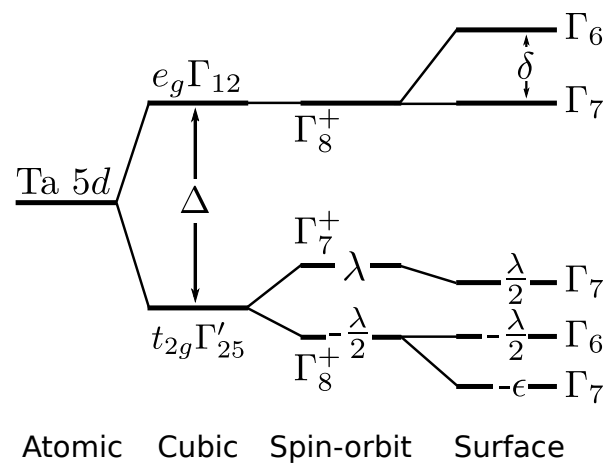

Figure 5: Level diagram of Ta- $d$ states at the $\Gamma$ point in the Brillouin zone, showing the effect of the crystal field, surface and the SOI. Rashba splitting occurs when both the SOI as well as broken inversion symmetry are present.

resides in the Ta- $d$ orbitals and the spin-orbit coupling in the oxygen is small, we can ignore the O- $p$ states for simplicity. Thus, the basis states consists of five $d$ orbitals times two spin states: $\phi_{\alpha} \equiv\left\{d_{z^{2} \uparrow \downarrow}, d_{x^{2}-y^{2} \uparrow \downarrow}, d_{x y \uparrow \downarrow}, d_{x z \uparrow \downarrow}, d_{y z \uparrow \downarrow}\right\}$. In this basis, the band structure term $H_{\mathrm{K}}$ with only nearest neighbor interactions can be written in the matrix form as,

$$
H_{\mathrm{K}}=\left(\begin{array}{ccccc}
h_{1} & h_{12} & 0 & 0 & 0 \\
h_{12}^{*} & h_{2} & 0 & 0 & 0 \\
0 & 0 & h_{3} & 0 & 0 \\
0 & 0 & 0 & h_{4} & 0 \\
0 & 0 & 0 & 0 & h_{5}
\end{array}\right) \otimes \sigma_{0}
$$

where $\otimes$ stands for the direct product and $\sigma_{0}$ is a $2 \times 2$ identity matrix. The 
nonzero matrix elements are,

$$
\begin{gathered}
h_{1}=\varepsilon_{1}+V_{\sigma} / 2\left(\cos k_{x}+\cos k_{y}\right) \\
h_{2}=\varepsilon_{2}+3 V_{\sigma} / 2\left(\cos k_{x}+\cos k_{y}\right) \\
h_{12}=-\sqrt{3} V_{\sigma} / 2\left(\cos k_{x}-\cos k_{y}\right) \\
h_{3}=\varepsilon_{3}+2 V_{\pi}\left(\cos k_{x}+\cos k_{y}\right) \\
h_{4}=\varepsilon_{4}+2 V_{\pi}\left(\cos k_{x}\right) \\
h_{5}=\varepsilon_{5}+2 V_{\pi}\left(\cos k_{y}\right)
\end{gathered}
$$

where $V_{\sigma}$ and $V_{\pi}$ are Slater-Koster overlap parameters $(d d \sigma)$ and $(d d \pi)$ respectively (the weakest $d d \delta$ overlap is ignored). The $\varepsilon_{i}$ are onsite energies taken as $\{\Delta+\delta, \Delta, \epsilon, 0,0\}$ from Fig. 5 .

The spin-orbit part $H_{\mathrm{SO}}$, in Eq. (1) only has onsite elements originating from $\lambda \mathbf{L} \cdot \mathbf{S}$ and no $\boldsymbol{k}$ dependence.

$$
H_{\mathrm{SO}}=\frac{i \lambda}{2}\left(\begin{array}{ccccc}
0 & 0 & 0 & -\sqrt{3} \sigma_{y} & \sqrt{3} \sigma_{x} \\
0 & 0 & -2 \sigma_{z} & \sigma_{y} & \sigma_{x} \\
0 & 2 \sigma_{z} & 0 & -\sigma_{x} & \sigma_{y} \\
\sqrt{3} \sigma_{y} & -\sigma_{y} & \sigma_{x} & 0 & -\sigma_{z} \\
-\sqrt{3} \sigma_{x} & -\sigma_{x} & -\sigma_{y} & \sigma_{z} & 0
\end{array}\right)
$$

where, $\lambda$ is the spin orbit coupling strength and $\sigma_{x}, \sigma_{y}, \sigma_{z}$ are the $2 \times 2$ Pauli spin matrices.

The third term $H_{\mathrm{E}}$ may originate from an external electric field or proximity to a surface that break the inversion symmetry of the system. In the simplest form, it can be written in terms of potential gradient $\eta$ as

$$
H_{\mathrm{E}}=\eta z .
$$

The broken symmetry gives rise to effective interactions between $d$ orbitals and can be separated into three distinct contributions as discussed in Ref. [20, $21,19]$ and briefly explained below. Adding up all the contributions gives the 
full matrix given below,

$$
H_{\mathrm{E}}=\left(\begin{array}{ccccc}
0 & 0 & 0 & h_{14} & h_{15} \\
0 & 0 & 0 & h_{24} & h_{25} \\
0 & 0 & 0 & h_{34} & h_{35} \\
h_{14}^{*} & h_{24}^{*} & h_{24}^{*} & 0 & 0 \\
h_{15}^{*} & h_{25}^{*} & h_{35}^{*} & 0 & 0
\end{array}\right)
$$

in which,

$$
\begin{gathered}
h_{14}=2 i \gamma_{1} \sin k_{x}, h_{15}=2 i \gamma_{1} \sin k_{y}, \\
h_{24}=2 i \gamma_{2} \sin k_{x}, h_{25}=-2 i \gamma_{2} \sin k_{y}, \\
h_{34}=2 i \gamma_{3} \sin k_{y}, h_{35}=2 i \gamma_{3} \sin k_{x} .
\end{gathered}
$$

where, $\gamma_{1}, \gamma_{2}$ and $\gamma_{3}$ are field induced overlap parameters. The three contributions that lead to the matrix above are discussed briefly below.

\subsubsection{Onsite contribution}

In the absence of external potential, the atomic orbitals $\left(\phi_{\alpha}\right)$ at each site $\left(\mathbf{R}_{i}\right)$ form an orthogonal set $\left|\phi_{\alpha}, \mathbf{R}_{i}\right\rangle$. However, these atomic wave functions are no longer orthogonal in the presence of external electric field and the matrix elements $\left\langle\phi_{\alpha}, \mathbf{R}_{i}\left|H_{\mathrm{E}}\right| \phi_{\beta}, \mathbf{R}_{i}\right\rangle$ are nonzero for orbitals that satisfy the selections rules $\Delta l= \pm 1$ and $\Delta m=0$ at the same site. [29] For $d$ orbitals, the selection rules are satisfied for $p$ and $f$ orbitals, which means that to incorporate this effect our basis states must include these orbitals. [24, 29]

Alternatively, one can treat $H_{\mathrm{E}}$ as perturbation and incorporate the couplings with $p$ and $f$ orbitals as first order corrections to the basis functions. [21] For example, the corrected $d_{z^{2}}$ orbital is,

$$
\left|d_{z^{2}}^{\prime}\right\rangle=\left|d_{z^{2}}\right\rangle+\frac{2 \eta_{p}}{\sqrt{15}}\left|p_{z}\right\rangle+\frac{3 \eta_{f}}{\sqrt{35}}\left|f_{z^{3}}\right\rangle
$$

where, $\eta_{p}$ and $\eta_{f}$ are parameters that depend on the orbital overlap, energy separation and electric field strength. [21] The atomic and perturbed $d_{z^{2}}$ orbitals for $\eta_{p}=0.58, \eta_{f}=0$ are shown in Fig. 6 . It confirms the intuitive picture 


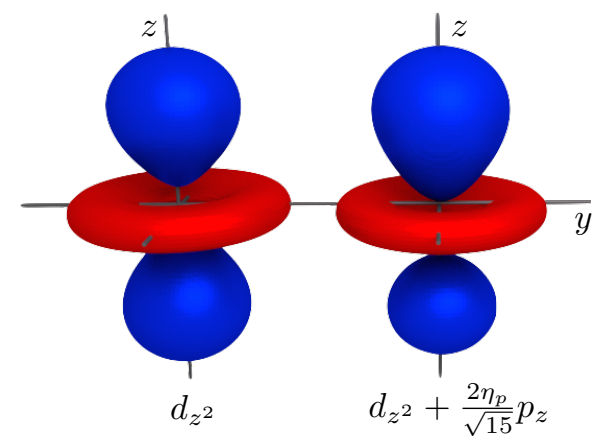

Figure 6: $3 \mathrm{D}$ isosurface plot of $3 d_{z^{2}}$ orbital showing the effect of mixing $p_{z}$ orbital as a consequence of symmetry breaking. The mixing factor $\eta_{p}$ is 0.58 . The colors indicate the sign of the wavefunction. The lobes becomes asymmetric due to the mixture.

that the electric field will make the orbitals asymmetric about the $x y$ plane, increasing the electron probability where potential energy is lower.

Finally, constructing the Hamiltonian in this basis introduces additional correction terms: $\left\langle\phi_{\alpha}\left|H_{\mathrm{K}}\right| \phi_{\beta}^{1}\right\rangle+\left\langle\phi_{\alpha}^{1}\left|H_{\mathrm{K}}\right| \phi_{\beta}\right\rangle$. When evaluated they lead to matrix elements given in Eq. 5 .

\subsubsection{Intersite contribution}

The electric field also leads to intersite terms of the type, $\eta\left\langle\phi_{\alpha}, \mathbf{R}_{i}|z| \phi_{\beta}, \mathbf{R}_{j}\right\rangle$ between orbitals that had no overlap before. These matrix elements can be evaluated using numerical integration or symmetry arguments. [21, 17] For $d$ orbitals on a square lattice, there are three unique parameters for intersite coupling:

$$
\begin{aligned}
& \xi_{1}=\eta\left\langle d_{z^{2}}|z| d_{x z}, a \hat{x}\right\rangle=\eta\left\langle d_{z^{2}}|z| d_{y z}, a \hat{y}\right\rangle, \\
& \xi_{2}=\eta\left\langle d_{x^{2}-y^{2}}|z| d_{x z}, a \hat{x}\right\rangle=\eta\left\langle d_{y z}|z| d_{x^{2}-y^{2}}, a \hat{y}\right\rangle, \\
& \xi_{3}=\eta\left\langle d_{x y}|z| d_{x z}, a \hat{y}\right\rangle=\eta\left\langle d_{x y}|z| d_{y z}, a \hat{x}\right\rangle .
\end{aligned}
$$

Here, the orbital on the left side of the inner product is assumed to be on the left atom and the orbital on the right sits on a neighbor shifted by the lattice constant $a$ along the indicated direction. Matrix elements arising from this contribution also have the form given in Eq. 5, when $\gamma_{i}$ are replaced with $\xi_{i}$. 


\subsubsection{Lattice contribution}

As discussed in the previous section, to screen the electric field, the lattice undergoes polar distortion in which the oxygen sublattice moves out of the metal plane inducing a dipole moment in the opposite direction. While this distortion significantly weakens the field, it also leads to new hopping matrix elements between metal $d$ and oxygen $p$ orbitals [19] as shown in Fig. 7. This contribution also requires $p$ orbitals to be included in the basis set for a full description. But, as with the onsite contribution, it can also be reduced to an effective $d-d$ coupling and has the form given in Eq. 5 .

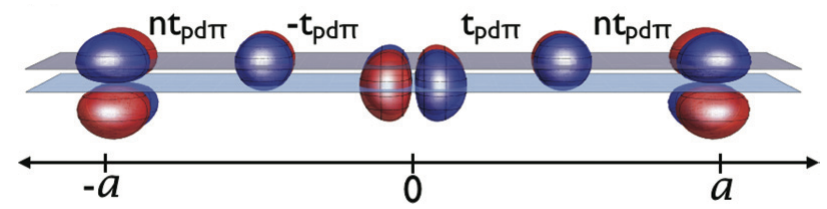

Figure 7: The $d$ orbitals in the transition metal ion $(-a, 0, a)$ and $p$ orbitals in oxygen $(-a / 2$, $a / 2$ ) in a distorted polar surface. Displacement of metal (light blue plane) and oxygen (light purple plane) sublattices are also shown. Lattice polarization leads to an effective hopping between $d_{x y}$ and $d_{x z}$ through $p_{x}$ orbitals. Figure taken from Ref. [19].

Combining Eqs. 2,3,5 we can construct the full Hamiltonian given in Eq. 1. Diagonalizing it at different $k$-points, we obtain the band structure shown in Fig. 8.

The case of small $\lambda \gg \varepsilon$ shown in Fig. 8 agrees well with the DFT bands shown in Fig. 1 for $\mathrm{TaO}_{2}$ surface. The figure also shows that the band splitting depends on the type of orbitals involved and the energy separation between them.

\subsection{Effective Hamiltonian}

Within perturbation theory, the Rashba splitting is a second order correction to the ground state energy. One can see this by inspecting the elements of the Hamiltonian matrix $H$ given in Eqs.(1-5). For example, consider the $d_{z^{2} \uparrow}, d_{z^{2} \downarrow}$ subspace: in the lowest order, the coupling between them is zero. However, $d_{z^{2} \uparrow}$ interacts with $d_{x z \uparrow}$ through the electric field term $2 i \gamma_{1} k_{x}\left(\sin k_{x} \approx k_{x}\right.$ in the limit of small $k$ ) and $d_{z^{2} \downarrow}$ interacts with $d_{x z \uparrow}$ through spin-orbit term $\sqrt{3} \lambda / 2$. 


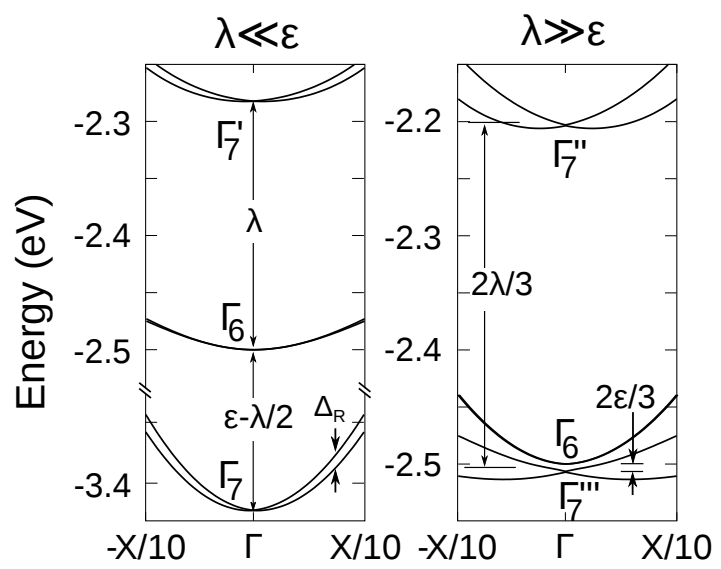

Figure 8: Rashba splitting of the $t_{2 g}$ states in the limit of weak and strong spin-orbit coupling $\lambda$ compared to the surface crystal field $\varepsilon$. The parameters are $\lambda=0.2, \varepsilon=1.0$ for left panel and $\lambda=0.2, \varepsilon=0.01$ for right panel. The electric field coupling parameters $\gamma=\{0,0,0.05\}$. All units are in $\mathrm{eV}$.

This leads to an effective coupling of the order $\sqrt{3} i \gamma_{1} \lambda k_{x} / \Delta$ between $d_{z^{2} \uparrow}$ and $d_{z^{2} \downarrow}$. Now, adding up contributions from $d_{x z \downarrow}, d_{y z \uparrow}$ and $d_{y z \downarrow}$ we get,

$$
\left\langle d_{z^{2} \uparrow}|H| d_{z^{2} \downarrow}\right\rangle=\frac{2 \sqrt{3} \gamma_{1} \lambda}{\Delta}\left(i k_{x}+k_{y}\right)
$$

which has the familiar Rashba form. This expression can also be derived more formally using the Löwdin downfolding method. [30] Thus, an effective Hamiltonian for $d_{z^{2}}$ band in the limit of weak $\lambda$ around $\Gamma$ point can be written as,

$$
\mathcal{H}_{z^{2}}(\Gamma)=\left(\begin{array}{cc}
\Delta+\delta+3 V_{\sigma}\left(1-\frac{k^{2}}{4}\right) & \frac{2 \sqrt{3} \lambda \gamma_{1}\left(i k_{x}+k_{y}\right)}{3 V_{\sigma}-4 V_{\pi}+\Delta+\delta} \\
\frac{2 \sqrt{3} \lambda \gamma_{2}\left(-i k_{x}+k_{y}\right)}{3 V_{\sigma}-4 V_{\pi}+\Delta+\delta} & \Delta+\delta+3 V_{\sigma}\left(1-\frac{k^{2}}{4}\right)
\end{array}\right)
$$

from which we get the Rashba parameter $\alpha_{R}=2 \sqrt{3} \gamma_{1} \lambda / \Delta$ when energy separation $\Delta$ is large. Similar expressions can be derived for all bands that are well separated from others.

\section{Summary}

DFT calculations on $\mathrm{KTaO}_{3}$ help us understand the Rashba effect in 2DEG systems. The calculations suggest that ionic relaxation weaken the polar field making the 2DEG less localized. The spin splitting is also diminished as a 


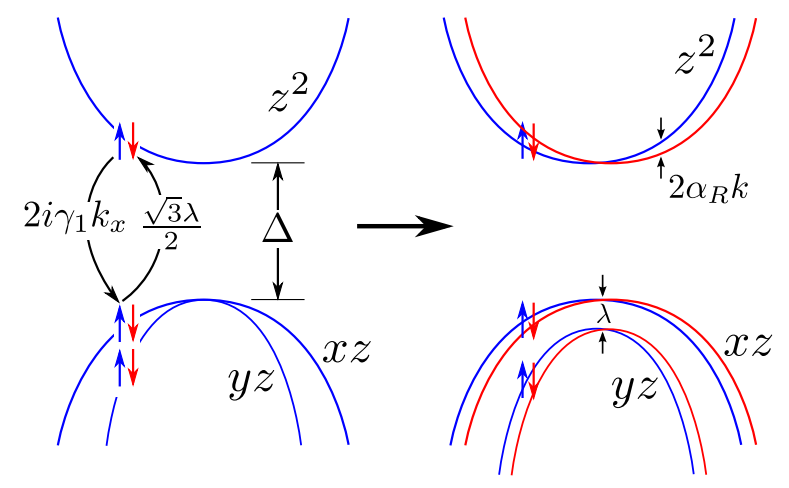

Figure 9: Rashba spin splitting through second-order perturbation theory. The bands shown in the left are spin degenerate. The $d_{z^{2} \uparrow}$ band interacts with $d_{x z \uparrow}$ through electric field $\left(2 i \gamma_{1} k_{x}\right)$ while the $d_{z^{2} \downarrow}$ band interacts $d_{x z \uparrow}$ through spin-orbit coupling $(\sqrt{3} \lambda / 2)$. Together, they cause splitting of the up and down bands as shown in the right. The curvature of $d_{x z}, d_{y z}$ bands are taken to be negative for clarity.

consequence. Rashba effect in this system is strongly orbital dependent and can be tuned by external factors such as gate voltages and strain. The steps involved in constructing a simple tight-binding model involving $d$ orbitals on a square lattice with inputs from DFT calculations are discussed. The electric field introduces new matrix elements in the Hamiltonian between $d$ orbitals by modifying the overlap, mixing with $p$ and $f$ states at the same sites as well as the distortion of metal-oxygen bonds. Thus, first principles and tightbinding methods offer powerful tools to complement experimental spectroscopic measurements of Rashba effect at polar surfaces and interfaces. With their help we can identify contributing factors and understand how to effectively control them.

\section{Acknowledgments}

The work done at MU was supported by the U. S. Department of Energy through Grant No. DE-FG02-00ER45818. The work at ORNL was supported by DOE, Basic Energy Sciences, Material Sciences and Engineering Division. The author wish to thank Sashi Satpathy and David Parker for useful discussions. 
[1] E. I. Rashba, Properties of semiconductors with an extremum loop .1. Cyclotron and combinational resonance in a magnetic field perpendicular to the plane of the loop, Sov. Phys. Solid. State 2 (1960) 1109.

[2] S. LaShell, B. McDougall, E. Jensen, Spin splitting of an au(111) surface state band observed with angle resolved photoelectron spectroscopy, Phys. Rev. Lett. 77 (16) (1996) 3419. doi:10.1103/physrevlett.77.3419.

URL http://dx.doi.org/10.1103/PhysRevLett.77.3419

[3] Y. Koroteev, G. Bihlmayer, J. Gayone, E. Chulkov, S. Blügel, P. Echenique, P. Hofmann, Strong spin-orbit splitting on bi surfaces, Phys. Rev. Lett. 93 (4) (2004) 046403. doi:10.1103/physrevlett.93.046403.

URL http://dx.doi.org/10.1103/PhysRevLett.93.046403

[4] K. Ishizaka, M. S. Bahramy, H. Murakawa, M. Sakano, T. Shimojima, T. Sonobe, K. Koizumi, S. Shin, H. Miyahara, A. Kimura, K. Miyamoto, T. Okuda, H. Namatame, M. Taniguchi, R. Arita, N. Nagaosa, K. Kobayashi, Y. Murakami, R. Kumai, Y. Kaneko, Y. Onose, Y. Tokura, Giant rashba-type spin splitting in bulk bitei, Nat. Mater. 10 (7) (2011) 521. doi:10.1038/nmat3051.

URL http://dx.doi.org/10.1038/nmat3051

[5] P. D. C. King, R. C. Hatch, M. Bianchi, R. Ovsyannikov, C. Lupulescu, G. Landolt, B. Slomski, J. H. Dil, D. Guan, J. L. Mi, E. D. L. Rienks, J. Fink, A. Lindblad, S. Svensson, S. Bao, G. Balakrishnan, B. B. Iversen, J. Osterwalder, W. Eberhardt, F. Baumberger, P. Hofmann, Large tunable rashba spin splitting of a two-dimensional electron gas in bi $_{2} \mathrm{se}_{3}$, Phys. Rev. Lett. 107 (9) (2011) 096802. doi:10.1103/physrevlett.107.096802.

URL http://dx.doi.org/10.1103/PhysRevLett.107.096802

[6] A. D. Caviglia, M. Gabay, S. Gariglio, N. Reyren, C. Cancellieri, J.-M. Triscone, Tunable rashba spin-orbit interaction at oxide interfaces, Phys. Rev. Lett. 104 (12) (2010) 126803. doi:10.1103/physrevlett.104.126803. URL http://dx.doi.org/10.1103/PhysRevLett.104.126803 
[7] M. Ben Shalom, M. Sachs, D. Rakhmilevitch, A. Palevski, Y. Dagan, Tuning spin-orbit coupling and superconductivity at the srtio $_{3} / \mathrm{laalo}_{3}$ interface: A magnetotransport study, Phys. Rev. Lett. 104 (12) (2010) 126802. doi:10.1103/physrevlett.104.126802.

URL http://dx.doi.org/10.1103/PhysRevLett.104.126802

[8] H. Nakamura, T. Koga, T. Kimura, Experimental evidence of cubic rashba effect in an inversion-symmetric oxide, Phys. Rev. Lett. 108 (20) (2012) 206601. doi:10.1103/physrevlett.108.206601.

URL http://dx.doi.org/10.1103/PhysRevLett.108.206601

[9] P. King, R. He, T. Eknapakul, P. Buaphet, S.-K. Mo, Y. Kaneko, S. Harashima, Y. Hikita, M. Bahramy, C. Bell, Z. Hussain, Y. Tokura, Z.-X. Shen, H. Hwang, F. Baumberger, W. Meevasana, Subband structure of a two-dimensional electron gas formed at the polar surface of the strong spin-orbit perovskite $\mathrm{ktaO}_{3}$, Phys. Rev. Lett. 108 (11) (2012) 117602. doi:10.1103/physrevlett.108.117602.

URL http://dx.doi.org/10.1103/PhysRevLett.108.117602

[10] S. Datta, B. Das, Electronic analog of the electro-optic modulator, Appl. Phys. Lett. 56 (7) (1990) 665. doi:10.1063/1.102730.

URL http://dx.doi.org/10.1063/1.102730

[11] Y. A. Bychkov, E. I. Rashba, Oscillatory effects and the magnetic susceptibility of carriers in inversion layers, J. Phys. C 17 (33) (1984) 6039. doi:10.1088/0022-3719/17/33/015.

URL http://dx.doi.org/10.1088/0022-3719/17/33/015

[12] Z. Popović, S. Satpathy, R. Martin, Origin of the two-dimensional electron gas carrier density at the laalo3 on srtio3 interface, Phys. Rev. Lett. 101 (25) (2008) 256801. doi:10.1103/physrevlett.101.256801.

URL http://dx.doi.org/10.1103/PhysRevLett.101.256801

[13] N. Nakagawa, H. Y. Hwang, D. A. Muller, Why some interfaces cannot be 
sharp, Nat. Mater. 5 (3) (2006) 204. doi:10.1038/nmat1569.

URL http://dx.doi.org/10.1038/nmat1569

[14] J. N. Eckstein, Oxide interfaces: Watch out for the lack of oxygen, Nat. Mater. 6 (7) (2007) 473. doi:10.1038/nmat1944.

URL http://dx.doi.org/10.1038/nmat1944

[15] P. Willmott, S. Pauli, R. Herger, C. Schlepütz, D. Martoccia, B. Patterson, B. Delley, R. Clarke, D. Kumah, C. Cionca, et al., Structural basis for the conducting interface between laalo3 and srtio3, Phys. Rev. Lett. 99 (15) (2007) 155502. doi:10.1103/physrevlett.99.155502.

URL http://dx.doi.org/10.1103/PhysRevLett.99.155502

[16] D. G. Schlom, J. Mannhart, Oxide electronics: Interface takes charge over si, Nat. Mater. 10 (3) (2011) 168. doi:10.1038/nmat2965.

URL http://dx.doi.org/10.1038/nmat2965

[17] Z. Zhong, A. Tóth, K. Held, Theory of spin-orbit coupling at laalo ${ }_{3} / \mathrm{srtio}_{3}$ interfaces and srtio $_{3}$ surfaces, Phys. Rev. B 87 (16) (2013) 161102. doi:10.1103/physrevb.87.161102.

URL http://dx.doi.org/10.1103/PhysRevB.87.161102

[18] Y. Kim, R. M. Lutchyn, C. Nayak, Origin and transport signatures of spinorbit interactions in one- and two-dimensional srtio 3 -based heterostructures, Phys. Rev. B 87 (24) (2013) 245121. doi:10.1103/physrevb.87.245121. URL http://dx.doi.org/10.1103/PhysRevB.87.245121

[19] G. Khalsa, B. Lee, A. H. MacDonald, Theory of $t_{2 g}$ electrongas rashba interactions, Phys. Rev. B 88 (4) (2013) 041302. doi:10.1103/physrevb.88.041302.

URL http://dx.doi.org/10.1103/PhysRevB.88.041302

[20] K. V. Shanavas, S. Satpathy, Electric field tuning of the rashba effect in the polar perovskite structures, Phys. Rev. Lett. 112 (8) (2014) 086802. 
doi:10.1103/physrevlett.112.086802.

URL http://dx.doi.org/10.1103/PhysRevLett.112.086802

[21] K. V. Shanavas, Z. Popović, S. Satpathy, Model hamiltonian for rashba spin-orbit interaction in $d$ electron solids, unpublished manuscript (2014).

[22] L. Petersen, P. Hedegård, A simple tight-binding model of spin-orbit splitting of sp-derived surface states, Surf. Sci. 459 (1-2) (2000) 49. doi:10.1016/s0039-6028(00)00441-6.

URL http://dx.doi.org/10.1016/S0039-6028(00)00441-6

[23] C. L. Kane, E. J. Mele, Quantum spin hall effect in graphene, Phys. Rev. Lett. 95 (22) (2005) 226801. doi:10.1103/physrevlett.95.226801.

URL http://dx.doi.org/10.1103/PhysRevLett.95.226801

[24] S. Konschuh, M. Gmitra, J. Fabian, Tight-binding theory of the spinorbit coupling in graphene, Phys. Rev. B 82 (24) (2010) 245412. doi:10.1103/physrevb.82.245412.

URL http://dx.doi.org/10.1103/PhysRevB.82.245412

[25] S. Caprara, F. Peronaci, M. Grilli, Intrinsic instability of electronic interfaces with strong rashba coupling, Physical Review Letters 109 (19) (2012) 196401. doi:10.1103/physrevlett.109.196401.

URL http://dx.doi.org/10.1103/PhysRevLett.109.196401

[26] G. Kresse, J. Hafner, Ab initio molecular dynamics for liquid metals, Phys. Rev. B 47 (1) (1993) 558. doi:10.1103/physrevb.47.558.

URL http://dx.doi.org/10.1103/PhysRevB.47.558

[27] G. Kresse, Efficient iterative schemes for ab initio total-energy calculations using a plane-wave basis set, Phys. Rev. B 54 (16) (1996) 11169. doi:10.1103/physrevb.54.11169.

URL http://dx.doi.org/10.1103/PhysRevB.54.11169

[28] G. F. Koster, J. O. Dimmock, R. G. Wheeler, H. Statz, The Properties of the Thirty-Two Point Groups, The MIT Press, 1963. 
[29] C. R. Ast, I. Gierz, sp-band tight-binding model for the bychkov-rashba effect in a two-dimensional electron system including nearest-neighbor contributions from an electric field, Phys. Rev. B 86 (8) (2012) 085105. doi:10.1103/physrevb.86.085105.

URL http://dx.doi.org/10.1103/PhysRevB.86.085105

[30] P.-O. Löwdin, A note on the quantum-mechanical perturbation theory, J. Chem. Phys. 19 (11) (1951) 1396. doi:10.1063/1.1748067.

URL http://dx.doi.org/10.1063/1.1748067 